\title{
Isogenies of Jacobians
}

\author{
Valeria Marcucci, Juan Carlos Naranjo and Gian Pietro Pirola
}

\begin{abstract}
By studying the infinitesimal variations of the Hodge structure and a generalization of the classical Babbage-Enriques-Petri theorem, we prove that the Jacobian variety of a generic element of a codimension $k$ subvariety of $\mathcal{M}_{g}$ is not isogenous to different Jacobian if $g>3 k+4$. We extend this result to $k=1, g \geqslant 5$ by using degeneration methods.
\end{abstract}

\section{Introduction}

Let $\mathcal{Z}$ be a subvariety of the moduli space $\mathcal{M}_{g}$ of complex smooth curves of genus $g$ of codimension $k>0$. We want to show that under certain numerical restrictions, the Jacobian of a generic element of $\mathcal{Z}$ is not isogenous to a distinct Jacobian. In other words, for a given $C$, generic in $\mathcal{Z}$, all curves of genus $g$ contained in the Jacobian $J C$ of $C$ are birationally equivalent. This is an extension of the theorem proved by Bardelli and Pirola (see [BP89]) for the whole $\mathcal{M}_{g}$ and can be seen as a Noether-Lefschetz locus problem for surfaces which are the product of two curves of the same genus (see Corollary 6.2). More precisely, our result is as follows.

Theorem 1.1. Let $\mathcal{Z} \subset \mathcal{M}_{g}$ be a codimension $k$ subvariety, with $k>0$. If $g>3 k+4$ (in particular $g>7$ ), then the Jacobian of a generic curve $C$ of $\mathcal{Z}$ is not isogenous to another Jacobian. The same is true for $k=1$ and $g \geqslant 5$.

Observe that the theorem fails for $k=1$ and $g=4$ : in this case $\mathcal{M}_{4}$ is a divisor in $\mathcal{A}_{4}$. Therefore, if in the Siegel upper space $\mathcal{H}_{4}$, we intersect the Jacobian locus $\mathcal{J}_{4}$ with its image $j\left(\mathcal{J}_{4}\right)$ by the action of a fixed isogeny $j$, we get a divisor in $\mathcal{M}_{4}$ where the Jacobian of a generic element is isogenous to a different Jacobian.

For $g>3 k+4$, our strategy is as follows: After a base change we have two families of smooth complex curves of genus $g$ on a base variety $W$, say $\pi: \mathcal{C} \longrightarrow W$ and $\pi^{\prime}: \mathcal{C}^{\prime} \longrightarrow W$, and a family of isogenies of the associated family of Jacobians, that is,

$$
\chi: J\left(\mathcal{C}^{\prime}\right) \longrightarrow J(\mathcal{C}) \text {. }
$$

This means that for $t \in W$, the map $\chi_{t}: J\left(C_{t}^{\prime}\right) \longrightarrow J\left(C_{t}\right)$ is an isogeny, where $C_{t}^{\prime}=\pi^{\prime-1}(t)$ and $C_{t}=\pi^{-1}(t)$.

Received 06 September 2014, accepted in final form 13 December 2015.

2010 Mathematics Subject Classification 14H40, 14H10, $14 \mathrm{~K} 02$

Keywords: isogenies, Jacobians, moduli of curves, moduli of abelian varieties

This journal is (C) Foundation Compositio Mathematica 2016. This article is distributed with Open Access under the terms of the Creative Commons Attribution Non-Commercial License, which permits non-commercial reuse, distribution, and reproduction in any medium, provided that the original work is properly cited. For commercial re-use, please contact the Foundation Compositio Mathematica.

Naranjo was partially supported by the Proyecto de Investigación MTM2012-38122-C03-02. Pirola was partially supported by Gnsaga and by MIUR PRIN 2012: Moduli, strutture geometriche e loro applicazioni. Part of this research was done during the visit of Pirola to the IMUB in the spring of 2013. 


\section{ISOGENIES OF JACOBIANS}

It follows that the associated rational Hodge structures are isomorphic. Consider the local (polarized) systems $\Lambda_{\mathbb{Z}}=R^{1} \pi_{*} \mathbb{Z}$ and $\Lambda_{\mathbb{Z}}^{\prime}=R^{1} \pi_{*}^{\prime} \mathbb{Z}$, and the systems $\Lambda_{\mathbb{C}}=R^{1} \pi_{*} \mathbb{C}$ and $\Lambda_{\mathbb{C}}^{\prime}=$ $R^{1} \pi_{*}^{\prime} \mathbb{C}$ obtained by tensoring by $\mathbb{C}$.

In particular, the infinitesimal variations of the Hodge structure associated with the Hodge filtrations of $\Lambda^{1,0} \subset \Lambda_{\mathbb{C}}$ and $\Lambda^{\prime 1,0} \subset \Lambda_{\mathbb{C}}^{\prime}$ are isomorphic. We borrowed this basic observation from Claire Voisin (see [BP89, Remark 4.2.5]). It is well known that the infinitesimal invariant of the Hodge structure of curves determines the quadrics that contain a canonical curve (see [CGGH83]). This allows us to translate our problem to a geometric one. Let $I(2)$ and $J(2)$ be the spaces of quadrics that contain the canonical curves associated with $C_{t}$ and $D_{t}$, respectively. It follows that under the choice of a suitable canonical embedding, $I(2) \cap J(2)$ has codimension at least $k$, where $k$ is the codimension of $m(W)$ in $\mathcal{M}_{g}$ and $m$ is the modular mapping $m: W \longrightarrow \mathcal{M}_{g}$. We can bound the codimension $k$ by using the Clifford index. For this we prove a result that gives an interesting (at least in our opinion) reconstruction result of the curve from a partial system of quadrics. It is a generalization of the Babbage-Enriques-Petri theorem (see, for example, [ACGH85, Chapter 3, Section 3]).

Theorem 1.2. Let $C$ be a curve of genus $g$ and Clifford index c. Let $I_{2} \subset \operatorname{Sym}^{2} H^{0}\left(C, \omega_{C}\right)$ be the vector space of the equations of the quadrics containing $C$, and let $K \subset I_{2}$ be a linear subspace of codimension $k$. If $g>2 k+5$ and $c>k+1$, then $C$ is the only irreducible non-degenerate curve contained in the intersection of the quadrics of $K$.

Corollary 1.3. Let $C$ be a generic curve in a codimension $k$ subvariety $\mathcal{Z}$ of $\mathcal{M}_{g}$. Let $I_{2} \subset$ $\operatorname{Sym}^{2} H^{0}\left(C, \omega_{C}\right)$ be the vector space of the equations of the quadrics containing $C$. Let $K \subset I_{2}$ be a linear subspace of codimension $k$. If $g>3 k+4$, then $C$ is the only irreducible non-degenerate curve contained in the intersection of the quadrics of $K$.

The corollary is a consequence of Theorem 1.2. Indeed, let $c$ be the Clifford index of a generic element of $\mathcal{Z}$. The locus of curves with given Clifford index can have several components and the minimal codimension is attained when $c$ is realized by a $g_{d}^{1}$ linear series, with $c=d-2$. This follows easily by a parameter count from a result in [CM91], where it is proved that a curve $C$ with Clifford index $c$ either is $(c+2)$-gonal or has Brill-Noether locus $W_{c+3}^{1}(C)$ of dimension at least 1.

Then, by the Riemann-Hurwitz theorem, the codimension of the component of the curves with a $g_{c+2}^{1}$ linear series is $3 g-3-(2 g-2+2(c+2)-3)=g-2 c-2$. Hence, since we assume $g>3 k+4$, we have

$$
k \geqslant g-2 c-2>3 k+4-2 c-2=3 k-2 c+2 .
$$

Therefore $c>k+1$ and the result follows from Theorem 1.2 since $g>3 k+4$ implies $g>2 k+5$ for $k>0$.

In Section 2 we start the proof of Theorem 1.1 under the hypothesis $g>3 k+4$ by reducing it to Corollary 1.3 following Voisin's observation indicated above. Theorem 1.2 will be proved in Section 3. The idea of the proof is as follows: Assuming the existence of a second non-degenerate curve in the intersection of the quadrics, we select linearly independent points $x_{i}$ on this curve. Then, by constructing a suitable rank 2 vector bundle on $C$, we are able to find points $p_{j} \in C$ such that the linear span of the points $x_{i}$ is contained in the linear span of the points $p_{j}$. From this it is easy to obtain a contradiction by using a theorem of Ran [Ran86].

To prove the divisorial case of the main theorem, we use the original approach in [BP89], 


\section{Marcucci, J. C. Naranjo and G. P. Pirola}

based on the analysis of the map

$$
\chi_{\mathbb{Z}}: \Lambda_{\mathbb{Z}} \longrightarrow \Lambda_{\mathbb{Z}}^{\prime}
$$

(in fact we will work with the dual lattices, that is, with the homology groups). If we can prove that $\chi_{\mathbb{Z}}\left(\Lambda_{\mathbb{Z}}\right)=n \Lambda_{\mathbb{Z}}^{\prime}$ for $n \in \mathbb{Z}$, we will get that $\mathcal{C}_{t}^{\prime}$ is isomorphic to $\mathcal{C}_{t}$ and $\chi_{t}$ is given by the multiplication by $n$. We use degeneration to $\Delta_{0}$ and study the monodromy action on $\Lambda_{\mathbb{Z}}$. The basic geometric information is now encoded on the generalized Jacobians. Roughly speaking, one has to prove that part of the limit map $\chi_{0}$ is the multiplication by $n$. This gives that the map $\chi_{t}$ is the multiplication by $n$ on part of the invariant cycles. Finally, we need to have degenerations with independent monodromy to complete the proof. It is clear that to follow this strategy one needs to control the degeneration type. Using the theory of divisors on $\mathcal{M}_{g}$ and following a valuable suggestion of Gavril Farkas, we realize the above program when $c=1$. The degeneration procedure is performed in Sections 4, 5 and 6. We will prove the existence of a type of degeneration (if $c=1$ ) to the union of a curve of genus $g-2$ and two generic elliptic tails. The independent degenerations to $\Delta_{0}$ are obtained by letting the elliptic tails become singular. To extract more information from the degeneration, we analyze the type of monodromy involved, that we classify in three cases (a, b, c of Section 5). Then we analyze the geometry of the generalized Jacobians by comparing their extension classes. In Section 6 we complete the proof by comparing the invariants of the two degenerations.

\section{Reduction to a problem on quadrics through the canonical curve}

The aim of this section is to prove that Corollary 1.3 implies Theorem 1.1 under the hypotheses $k>0$ and $g>3 k+4$.

Remember that an isogeny $\chi: A^{\prime} \longrightarrow A$ between principally polarized Abelian varieties $\left(A^{\prime}, L_{A^{\prime}}\right)$ and $\left(A, L_{A}\right)$ such that $\chi^{*} L_{A} \cong L_{A^{\prime}}^{\otimes m}$ is determined by a subgroup $H$ of the group of $m$-torsion points $A_{m}^{\prime}$ totally isotropic with respect to the Riemann bilinear form

$$
e_{m}: A_{m}^{\prime} \times A_{m}^{\prime} \longrightarrow \mu_{m}
$$

$\left(A=A^{\prime} / H\right)$ and a level subgroup $\tilde{H}$ of the theta group $\mathcal{G}\left(L_{A^{\prime}}^{\otimes m}\right)$; see [Mum70, Chapter 23]. Then the moduli space of those isogenies can be rewritten as

$$
\widetilde{\mathcal{A}}_{g}^{m}=\left\{\chi: A^{\prime} \longrightarrow A, \chi^{*}\left(L_{A}\right) \cong L_{A^{\prime}}^{\otimes m}\right\} / \cong=\left\{\left(A^{\prime}, L_{A^{\prime}} ; H, \tilde{H}\right)\right\} / \cong
$$

and the forgetful map is a finite covering $\varphi: \widetilde{\mathcal{A}}_{g}^{m} \longrightarrow \mathcal{A}_{g}$. Moreover, the map $\psi: \widetilde{\mathcal{A}}_{g}{ }^{m} \longrightarrow \mathcal{A}_{g}$ sending $\chi: A^{\prime} \longrightarrow A$ to $\left(A, L_{A}\right)$ is another covering space.

Given a generic isogeny $\chi: A^{\prime} \longrightarrow A$, we consider tangent spaces in the following diagram:

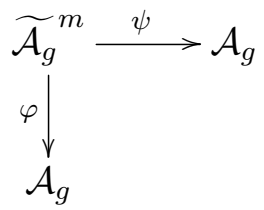


and we get an isomorphism $\lambda$ as follows:

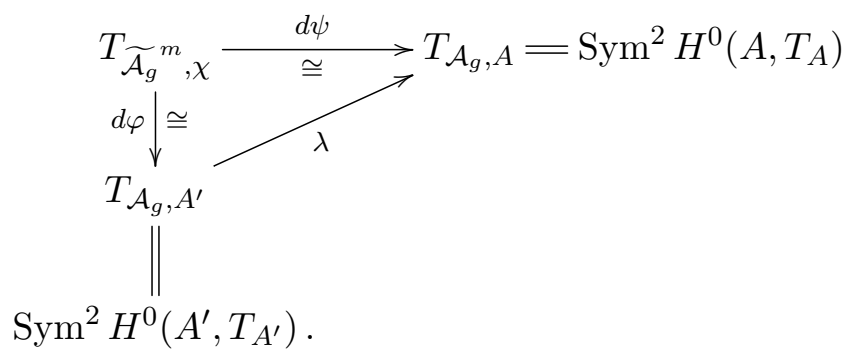

Coming back to our problem, let us assume that the locus of curves in $\mathcal{M}_{g}$ with Jacobian isogenous to the Jacobian of some curve in $\mathcal{Z}$ contains a codimension $k$ component $\mathcal{Z}^{\prime} \subset \mathcal{M}_{g}$. Our hypothesis on $k$ implies that a generic element $C^{\prime} \in \mathcal{Z}^{\prime}$ satisfies $\operatorname{End}(J C) \cong \mathbb{Z}$ (see [CGT92] or [Pir88]). Therefore an isogeny $\chi: J C^{\prime} \longrightarrow J C$ must satisfy that the pull-back of the principal polarization in $J C$ is a multiple of the principal polarization in $J C^{\prime}$. Hence there exist an integer $m$ and an irreducible variety $\mathcal{R} \subset{\widetilde{\mathcal{A}_{g}}}^{m}$ dominating $\mathcal{Z}^{\prime}$ and $\mathcal{Z}$ through $\varphi$ and $\psi$, respectively. Set $\mathcal{M}:=\varphi^{-1}\left(\mathcal{M}_{g}\right)$ and $\mathcal{M}^{\prime}:=\psi^{-1}\left(\mathcal{M}_{g}\right)$. Then $\mathcal{R} \subset \mathcal{M} \cap \mathcal{M}^{\prime}$. Fix a generic element $\chi: J C^{\prime} \longrightarrow J C$ in $\mathcal{R}$. In the following diagram we consider in the first row the natural inclusions of tangent spaces at $\chi$ and we put in the second row the first row's image by $d \varphi$ :

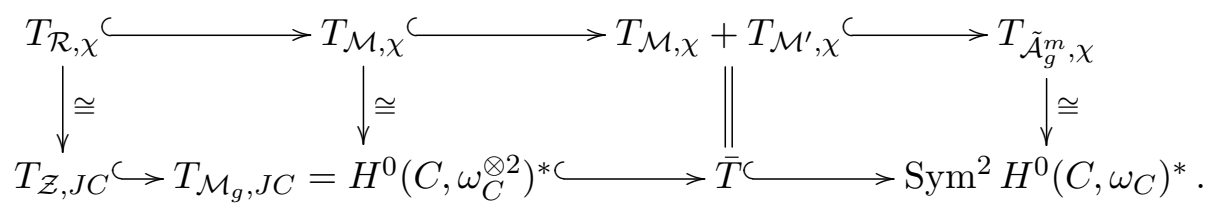

Observe that, by the Grassmann formula, the dimension of $\bar{T}$ is at most $3 g-3+k$. Set $K(C):=\operatorname{Ker}\left(\operatorname{Sym}^{2} H^{0}\left(C, \omega_{C}\right) \longrightarrow \bar{T}^{*}\right)$; this is a subspace of the vector space $I_{2}(C)$ of the quadrics containing the image of $C$ by the canonical map. The codimension of $K(C)$ in $I_{2}(C)$ is at most $k$. By using $\psi$ instead of $\varphi$ we get the corresponding vector space $K\left(C^{\prime}\right) \subset I_{2}\left(C^{\prime}\right)$ and we obtain a canonical isomorphism $K(C) \cong K\left(C^{\prime}\right)$. Then Corollary 1.3 implies that $C$ and $C^{\prime}$ are isomorphic and, since $\operatorname{End}(J C)=\mathbb{Z}$, the isogeny is a multiple of the identity.

\section{A generalization of the Babbage-Enriques-Petri theorem}

This section is devoted to the proof of Theorem 1.2. We fix the notation $K \subset I_{2}$ of the statement. We assume that the intersection of all the quadrics of $K$ contains an irreducible non-degenerate curve different from $C$. In particular, we can select $k+1$ linearly independent points $x_{i} \in$ $\bigcap_{Q \in K} Q \subset \mathbb{P} H^{0}\left(C, \omega_{C}\right)^{*}$ such that $x_{i} \notin C$. We choose a representative of $x_{i}$ in $H^{0}\left(C, \omega_{C}\right)^{*}$ and we denote it by the same symbol. Then $x_{i} \otimes x_{i} \in \operatorname{Sym}^{2} H^{0}\left(C, \omega_{C}\right)^{*}$. We denote by $L$ the linear variety spanned by these points.

Let $R$ and $R^{\prime}$ be the quotients $I_{2} / K$ and $\operatorname{Sym}^{2} H^{0}\left(C, \omega_{C}\right) / K$, respectively. Then we have the 
diagram of vector spaces

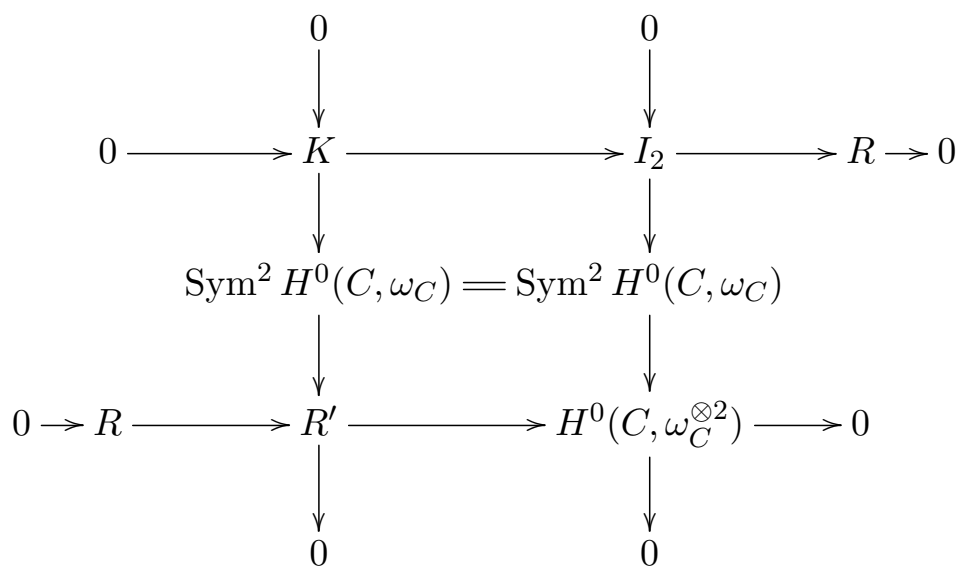

and its dual

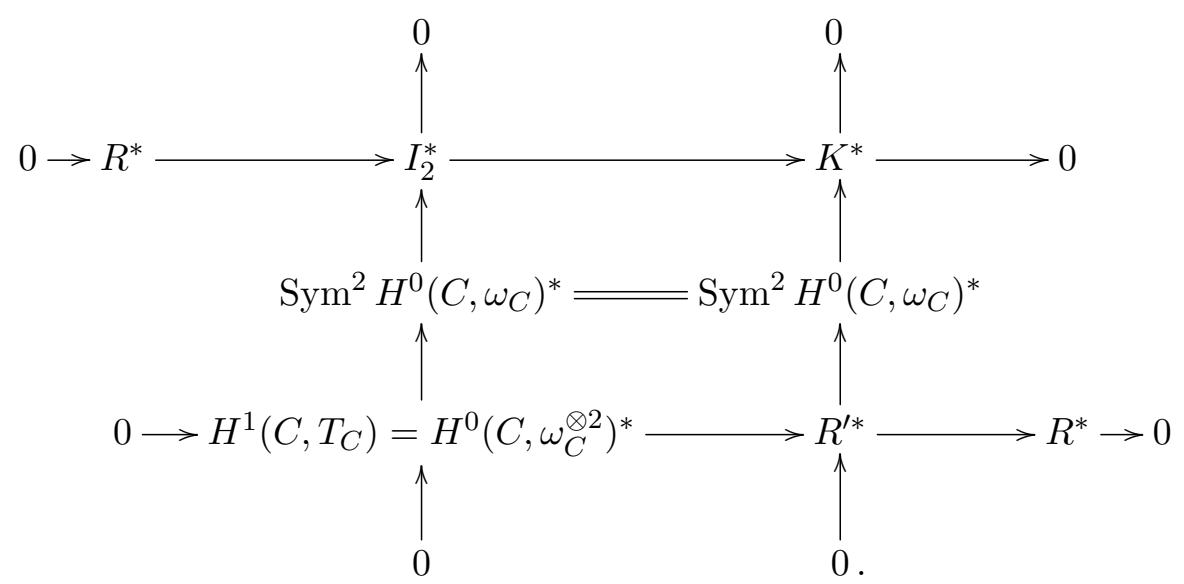

Since all the quadrics of $K$ vanish at $x_{i}$, the image of $L$ in $K^{*}$ is zero, hence $L \subset R^{\prime *}$. Since $L$ has dimension $k+1$ and $\operatorname{dim} R=k$ by the hypothesis on $K$, we have $H^{1}\left(C, T_{C}\right) \cap L \neq(0)$. Let $\alpha$ be a non-trivial element in this intersection. Looking at $H^{1}\left(C, T_{C}\right)=\operatorname{Ext}^{1}\left(\omega_{C}, \mathcal{O}_{C}\right)$ as classes of extensions, we associate with $\alpha$ a rank 2 vector bundle $E_{\alpha}$ and a short exact sequence:

$$
0 \longrightarrow \mathcal{O}_{C} \longrightarrow E_{\alpha} \longrightarrow \omega_{C} \longrightarrow 0 \text {. }
$$

The coboundary map $H^{0}\left(C, \omega_{C}\right) \longrightarrow H^{1}\left(C, \mathcal{O}_{C}\right)$ is the cup-product with $\alpha$. Since $\alpha \in L$, we have $\alpha=\sum_{i=1}^{k+1} a_{i} x_{i} \otimes x_{i}$. Therefore, if we denote by $H_{i}$ the kernel of the form $x_{i}: H^{0}\left(C, \omega_{C}\right) \longrightarrow \mathbb{C}$, the intersection $H_{1} \cap \cdots \cap H_{k+1}$ is contained in $\operatorname{Ker}(\cdot \cup \alpha)$; in fact,

$$
\operatorname{Ker}(\cdot \cup \alpha)=\bigcap_{i \text { with } a_{i} \neq 0} H_{i}
$$

We can assume that $x_{1}, \ldots, x_{k^{\prime}}$ for $k^{\prime} \leqslant k+1$ are the points such that $a_{i} \neq 0$. Then there are $g-k^{\prime}$ sections of $H^{0}\left(C, \omega_{C}\right)$ lifting to $E_{\alpha}$. Let $W \subset H^{0}\left(C, E_{\alpha}\right)$ be the vector space generated by these sections. We consider the wedge product of sections

$$
\psi: \Lambda^{2} W \hookrightarrow \Lambda^{2} H^{0}\left(C, E_{\alpha}\right) \longrightarrow H^{0}\left(C, \operatorname{det} E_{\alpha}\right)=H^{0}\left(C, \omega_{C}\right) .
$$

The hypothesis $g>2 k+5$ implies that the projectivization of the kernel of $\psi$ (which has codimension at most $g$ ) intersects, in $\mathbb{P}\left(\Lambda^{2} W\right)$, the Grassmannian of the decomposable elements. 


\section{ISOGENIES OF JACOBIANS}

Hence there are two sections $s_{1}, s_{2} \in W \subset H^{0}\left(C, E_{\alpha}\right)$ such that $s_{1} \wedge s_{2}=0$. This means that they generate a rank 1 torsion-free sheaf $M_{\alpha} \subset E_{\alpha}$, hence a line bundle. By construction $h^{0}\left(C, M_{\alpha}\right) \geqslant 2$. Let us consider the quotient of sheaves $Q_{\alpha}=E_{\alpha} / M_{\alpha}$, and let $Q_{\alpha}^{0}$ be the quotient of $Q_{\alpha}$ by its torsion subsheaf. The kernel $L_{\alpha}$ of the natural map $E_{\alpha} \longmapsto Q_{\alpha}^{0}$ is a line bundle that by construction contains $M_{\alpha}$. In particular, $h^{0}\left(C, L_{\alpha}\right) \geqslant 2$. Observe that $Q_{\alpha}^{0} \cong \omega_{C} \otimes L_{\alpha}^{-1}$. We get a diagram

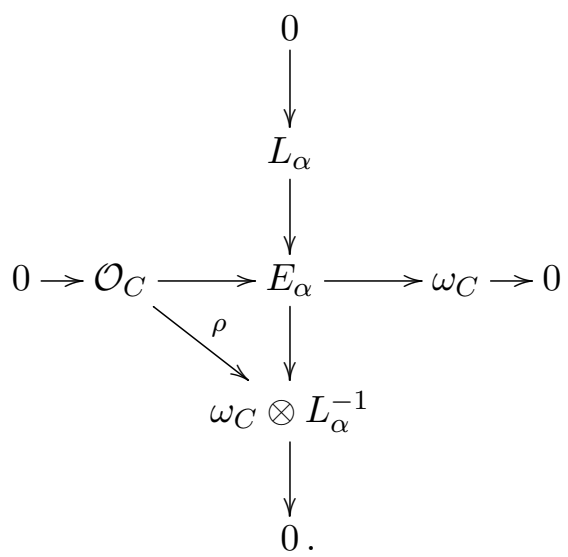

Note that $\rho \neq 0$, otherwise the section of $E_{\alpha}$ represented by the horizontal arrow $\mathcal{O} \longrightarrow E_{\alpha}$ would belong to $W$, which contradicts the definition of $W$.

Observe that the existence of the map $\rho$ implies that $h^{0}\left(C, \omega_{C} \otimes L_{\alpha}^{-1}\right)$ is positive. We distinguish two cases.

Case 1: $h^{0}\left(C, \omega_{C} \otimes L_{\alpha}^{-1}\right) \geqslant 2$. Then we can use $L_{\alpha}$ to compute the Clifford index of the curve. We have

$$
h^{0}\left(C, L_{\alpha}\right)+h^{0}\left(C, \omega_{C} \otimes L_{\alpha}^{-1}\right) \geqslant h^{0}\left(C, E_{\alpha}\right) \geqslant g-k^{\prime}+1,
$$

that combined with the Riemann-Roch theorem gives $2 h^{0}\left(C, L_{\alpha}\right) \geqslant \operatorname{deg}\left(L_{\alpha}\right)+2-k^{\prime}$. Therefore

$$
\operatorname{deg}\left(L_{\alpha}\right)-2 h^{0}\left(C, L_{\alpha}\right)+2 \leqslant k^{\prime} \leqslant k+1,
$$

which gives a contradiction since $k+1<c$ by hypothesis.

Case 2: $h^{0}\left(C, \omega_{C} \otimes L_{\alpha}^{-1}\right)=1$. Then $h^{0}\left(C, L_{\alpha}\right) \geqslant g-k^{\prime}$. Let $e$ be the degree of $\omega_{C} \otimes L_{\alpha}^{-1} \cong$ $\mathcal{O}_{C}\left(p_{1}+\cdots+p_{e}\right)$. We claim that $e \leqslant k^{\prime}$.

Indeed, as $\rho$ induces an isomorphism $H^{0}\left(C, \mathcal{O}_{C}\right) \cong H^{0}\left(C, \omega_{C} \otimes L_{\alpha}^{-1}\right)$, the map $H^{0}\left(C, E_{\alpha}\right) \longrightarrow$ $H^{0}\left(C, \omega_{C} \otimes L_{\alpha}^{-1}\right)$ is surjective and we get

$$
g-k^{\prime} \leqslant h^{0}\left(C, L_{\alpha}\right)=h^{0}\left(C, \omega_{C} \otimes L_{\alpha}^{-1}\right)+2 g-2-e+1-g=g-e ;
$$

the claim follows. Coming back to diagram (3.2), we obtain

$$
H^{0}\left(C, L_{\alpha}\right)=H^{0}\left(C, \omega_{C}\left(-p_{1}-\cdots-p_{e}\right)\right) \subset \operatorname{Ker}(\cdot \cup \alpha)=\bigcap_{i=1, \ldots, k^{\prime}} H_{i} .
$$

By dualizing, we obtain the inclusion of linear spans

$$
\left\langle x_{1}, \ldots, x_{k^{\prime}}\right\rangle \subset\left\langle p_{1}, \ldots, p_{e}\right\rangle .
$$

We denote by $\widetilde{C}_{0}$ a non-degenerate irreducible curve, $\widetilde{C}_{0} \neq C$, contained in all the quadrics parametrized by $K$. Let $\widetilde{C}$ be the normalization of $\widetilde{C}_{0}$, and let $\gamma$ be the normalization map. By 


\section{Marcucci, J. C. Naranjo and G. P. Pirola}

choosing generically the $k+1$ points $x_{i} \in \widetilde{C}$, we can assume that $k^{\prime}$ and $e$ are constant, so the correspondence

$$
\Gamma=\left\{\left(x_{1}+\cdots+x_{k^{\prime}}, p_{1}+\cdots+p_{e}\right) \mid\left\langle\gamma\left(x_{1}\right), \ldots, \gamma\left(x_{k^{\prime}}\right)\right\rangle \subset\left\langle p_{1}, \ldots, p_{e}\right\rangle\right\} \subset \widetilde{C}^{\left(k^{\prime}\right)} \times C^{(e)}
$$

dominates $\widetilde{C}^{\left(k^{\prime}\right)}$. Moreover, since $\widetilde{C}$ is non-degenerate, the fibers of $\pi_{2}: \Gamma \longrightarrow C^{(e)}$ must be finite. Since $e \leqslant k^{\prime} \leqslant \operatorname{dim} \Gamma=\operatorname{dim} \pi_{2}(\Gamma) \leqslant e$, we obtain $e=k^{\prime}$. On the other hand, the natural rational maps

$$
\begin{aligned}
& C^{(e)} \rightarrow \operatorname{Sec}^{e}(C) \subset \operatorname{Grass}\left(e-1, \mathbb{P}^{g-1}\right), \\
& \widetilde{C}^{(e)} \longrightarrow \operatorname{Sec}^{e}\left(\widetilde{C}_{0}\right) \subset \operatorname{Grass}\left(e-1, \mathbb{P}^{g-1}\right)
\end{aligned}
$$

are generically injective by the uniform position theorem (remember that $e=k^{\prime} \leqslant k+1$ and $2 k+5<g)$, hence the correspondence $\Gamma$ is of bidegree $(1,1)$ and therefore $\widetilde{C}^{(e)}$ and $C^{(e)}$ are birational. In particular, $g(\widetilde{C})=g(C)=g$ (the induced map on Jacobians $J \widetilde{C} \longrightarrow J C$ has to be dominant since the image generates and the same holds in the opposite direction). By Ran's theorem on symmetric products, see [Ran86], we get $C \cong \widetilde{C}$. Observe that both curves have to be canonical, hence $\widetilde{C}_{0}$ is smooth and there is a linear projective transformation $\varphi: \widetilde{C} \longmapsto C$. Coming back to our argument and choosing $k$ generic points $x_{i} \in \widetilde{C}$, we have $\left\langle x_{1}, \ldots, x_{k}\right\rangle=$ $\left\langle\varphi\left(x_{1}\right), \ldots, \varphi\left(x_{k}\right)\right\rangle$, hence $\varphi$ leaves $\operatorname{Sec}^{k}(C)$ invariant and must be the identity, so $\widetilde{C}=C$, which gives a contradiction.

\section{Divisor case, intersection with the boundary}

Now we start the proof of the codimension 1 case of the main theorem assuming $g \geqslant 5$. We write $\mathcal{D}$ instead of $\mathcal{Z}$. The initial step of our degeneration procedure is to show that the intersection of $\mathcal{D}$ with the boundary contains appropriate stable curves. These curves have to contain enough information to deduce from them the main result for the general smooth curve. The goal of this section is to define a family of convenient reducible curves and to prove that they appear in the closure of $\mathcal{D}$.

We start by recalling the following well-known facts on the rational Picard group of the compactified moduli space $\overline{\mathcal{M}}_{g}$ of stable curves (see for instance [ACG11]):

$$
\operatorname{Pic}_{\mathbb{Q}} \mathcal{M}_{g}=\lambda \mathbb{Q},
$$

where $\lambda$ is the Hodge class. Moreover,

$$
\overline{\mathcal{M}_{g}} \backslash \mathcal{M}_{g}=\bigcup_{i=0}^{\left[\frac{g-1}{2}\right]} \Delta_{i}
$$

and

$$
\operatorname{Pic}_{\mathbb{Q}}\left(\overline{\mathcal{M}_{g}}\right)=\left\langle\lambda, \delta_{0}, \delta_{1}, \ldots, \delta_{\left[\frac{g-1}{2}\right]}\right\rangle \mathbb{Q},
$$

where $\delta_{i}$ for $i>0$ is the class of the divisor $\Delta_{i}$ whose general point represents a nodal curve $C_{1} \cup C_{2}$, where $C_{1}$ and $C_{2}$ are integral, smooth curves of genus $i$ and $g-i$, respectively, intersecting in one point. And $\delta_{0}$ is the class of $\Delta_{0}$ whose general point represents an irreducible curve with exactly one node.

We denote by $d$ the class of $\overline{\mathcal{D}}$ in the rational Picard group. Then we can write

$$
d=a \lambda+\Sigma_{i \geqslant 0} a_{i} \delta_{i} .
$$


Remark 4.1. We note that $a$ must be different from zero. Otherwise the class of $\mathcal{D}$ in the rational Picard group of $\mathcal{M}_{g}$ would be zero. Since the Satake compactification $\overline{\mathcal{M}}_{g}^{s}$ of $\mathcal{M}_{g}$ is a projective variety and the boundary $\overline{\mathcal{M}}_{g}^{s}-\mathcal{M}_{g}$ has codimension 2 , for a smooth point $p$ of $\mathcal{D}$, there exists a complete curve $C$ in $\mathcal{M}_{g}$ going through $p$ and cutting $\mathcal{D}$ transversally. Hence $C \cdot \mathcal{D} \neq 0$, which gives a contradiction.

Now we consider a complete integral curve $B$ in $\mathcal{M}_{g-2}$ (it exists because $g-2 \geqslant 3$ ), and we fix two elliptic curves $E_{1}, E_{2}$ with arbitrary $j$-invariants $j_{1}, j_{2} \in \mathcal{M}_{1}$. Denote by $\Gamma_{b}$ the smooth curve of genus $g-2$ corresponding to $b$. We consider the set of the stable curves obtained by glueing the two elliptic curves to $\Gamma_{b}$ at two distinct points $p_{1}$ and $p_{2}$ of $\Gamma_{b}$. This does not depend on the choice of the points on the elliptic curves. This family is parametrized by the symmetric product $\Gamma_{b}^{(2)} \backslash \Delta_{\Gamma_{b}}$ minus the diagonal.

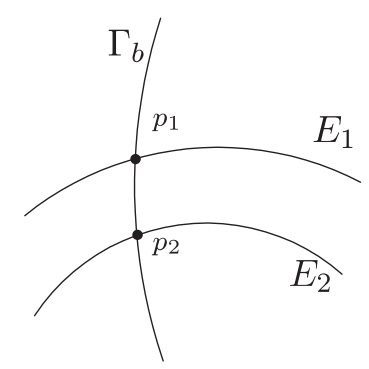

So we have a well-defined map

$$
\begin{aligned}
\Gamma_{b}^{(2)} \backslash \Delta_{\Gamma_{b}} & \longrightarrow \Delta_{1} \subset \overline{\mathcal{M}_{g}} \\
p_{1}+p_{2} & \longmapsto E_{1} \cup_{p_{1}} \Gamma \cup_{p_{2}} E_{2}
\end{aligned}
$$

that extends to the whole symmetric product by sending $2 p$ to the following curve: glue the infinity point of a $\mathbb{P}^{1}$ to the point $p$ and then glue $E_{1}$ and $E_{2}$ to two other points on the line. We note that these curves also belong to $\Delta_{2}$.

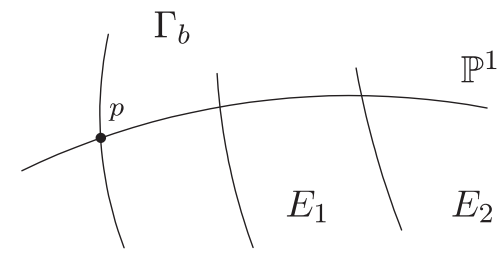

Finally, by moving $b$ in the curve $B$, we obtain a complete threefold $T \subset \Delta_{1}$. In other words, this threefold can be seen as the image in $\overline{\mathcal{M}_{g}}$ of the relative symmetric product over $B$ :

$$
T=\bigcup_{b \in B} \Gamma_{b}^{(2)}
$$

Our aim is to study the restriction of the divisor $\overline{\mathcal{D}}$ to $T$. To do this we do a computation in $\operatorname{Pic}_{\mathbb{Q}}(T)$. Denote by $S$ the surface in $T$ obtained as the union of all the diagonals:

$$
S=\bigcup_{b \in B} \Delta_{\Gamma_{b}}
$$

We will need the following vanishing results. 


\section{Marcucci, J. C. Naranjo and G. P. Pirola}

Lemma 4.2. The restriction of the class $\delta_{1}$ to $S$ is zero: $\left.\delta_{1}\right|_{S}=0$.

Proof. We fix a smooth curve $C$ of genus 2 with a marked point $x$. We glue $C$ to $\Gamma_{b}$, identifying $x$ with $p \in \Gamma_{b}$. Then, by moving $p$ in $\Gamma_{b}$ and $b$ in $B$, we construct an algebraic surface $S_{C}$ such that $\Delta_{1} \cap S_{C}=\emptyset$. Therefore $\delta_{1} \cdot S_{C}=0$. Now we degenerate $C$ to a genus 2 curve with a marked point consisting of the two elliptic curves $E_{1}$ and $E_{2}$ glued to a $\mathbb{P}^{1}$ at 0 and 1 , respectively, with $\infty$ being the marked point. Therefore, by adding the curve $\Gamma_{b}$ identifying $\infty$ with $p$, we get our surface $S$ as a limit of a family of algebraic surfaces $S_{C}$ as above. We obtain $\delta_{1} \cdot S=0$.

Lemma 4.3. For each $b \in B$, we have $\left.\lambda\right|_{\Delta_{\Gamma_{b}}}=0$.

Proof. The Hodge structure is constant along the diagonal.

We will also use the following basic observation.

Lemma 4.4. Let $N$ be a complete curve in $\overline{\mathcal{M}}_{g}$. Then $\left.\rho\right|_{N} \neq 0$ for at least one class $\rho \in$ $\left\{\lambda, \delta_{0}, \ldots, \delta_{\left[\frac{g-1}{2}\right]}\right\}$.

The main result of this section is the following.

Proposition 4.5. The restriction $\left.d\right|_{T}$ is not a multiple of the class of $S$ in $\operatorname{Pic}_{\mathbb{Q}}(T)$; that is, $\left.d\right|_{T} \neq m S$ for all $m \in \mathbb{Q}$. In particular, $\overline{\mathcal{D}} \cap T \neq \emptyset$ and this intersection contains elements outside $S$.

Proof. We use the notation introduced in (4.1). For a contradiction, assume $\left.d\right|_{T}=m S$. Notice that $\Delta_{i}$ does not intersect $T$ for $i=0$ and $i \geqslant 3$ and that $\Delta_{2} \cap T=S$, so we get

$$
\left.d\right|_{T}=m S=\left.a \lambda\right|_{T}+\left.a_{1} \delta_{1}\right|_{T}+a_{2} k S
$$

for some $k$. Therefore

$$
\left(m-a_{2} k\right) S=\left.a \lambda\right|_{T}+\left.a_{1} \delta_{1}\right|_{T} .
$$

Restricting to one diagonal $\Delta_{\Gamma_{b}}$ and using Lemmas 4.2 and 4.3 , we deduce that $m-a_{2} k=0$. Restricting now to $S$, we get $a \lambda \mid T=0$. Since $\lambda$ is not trivial on $T$, we obtain $a=0$, which contradicts Remark 4.1.

Remark 4.6. Observe that the isomorphism classes of $E_{1}$ and $E_{2}$ are arbitrary, hence they could represent the infinity class. Then the limit curves we were looking for are

$$
\mathcal{L}=\left\{E_{1} \cup_{p_{1}} \Gamma \cup_{p_{2}} E_{2} \in \overline{\mathcal{D}} \mid \Gamma \in \mathcal{M}_{g-2}, p_{1} \neq p_{2}, E_{1}, E_{2} \in \overline{\mathcal{M}_{1}}\right\} \subset \overline{\mathcal{D}} .
$$

We recall that a generic point of $M_{g}$, for $g \geqslant 3$, is contained in a complete curve (see Remark 4.1). Then the following result is a consequence of Proposition 4.5.

Corollary 4.7. There is a subvariety $\mathcal{R} \subset \mathcal{M}_{g-2}$ of codimension at most 1 such that for any $\Gamma \in \mathcal{R}$ and any $E_{1}, E_{2} \in \overline{\mathcal{M}_{1}}$, there are curves $E_{1} \cup_{p_{1}} \Gamma \cup_{p_{2}} E_{2}$ in $\mathcal{L}$.

Observe that all these elements belong to $\Delta_{1}$, and that they belong to $\Delta_{0}$ if and only if at least one of the elliptic curves represents the infinity class.

Remark 4.8. With the same techniques one can prove that the divisor $\overline{\mathcal{D}}$ contains irreducible curves with only one node. Moreover, by considering a surface in $\Delta_{i}$, for $i>0$, given by fixed smooth curves of genus $g-i$ and $i$, and moving the intersection point, one also shows with the same procedure that the divisor contains "generic" elements of $\Delta_{i}$. We do not use these facts in the rest of the paper. 


\section{ISOGENIES OF JACOBIANS}

\section{Limits of isogenies}

As in Section 2, we assume the existence of isogenies $J C^{\prime} \longrightarrow J C$ for generic elements $C \in \mathcal{D}$. In order to glue all these maps together to provide a family $\chi: \mathcal{J}^{\prime} \longrightarrow \mathcal{J}$, we need to pull back to a suitable branched cover of our divisor. Since our calculations will be mainly of local nature, we still denote this space of parameters by $\mathcal{D}$. Our goal is to get as much information as possible from the specialization of the family of isogenies to the curves of $\mathcal{L}$.

Let $L=E_{1} \cup_{p_{1}} \Gamma \cup_{p_{2}} E_{2} \in \mathcal{L} \subset \overline{\mathcal{D}}$ be a fixed limit curve (see Remark 4.6 and Corollary 4.7). We assume that one of the elliptic curves has a node.

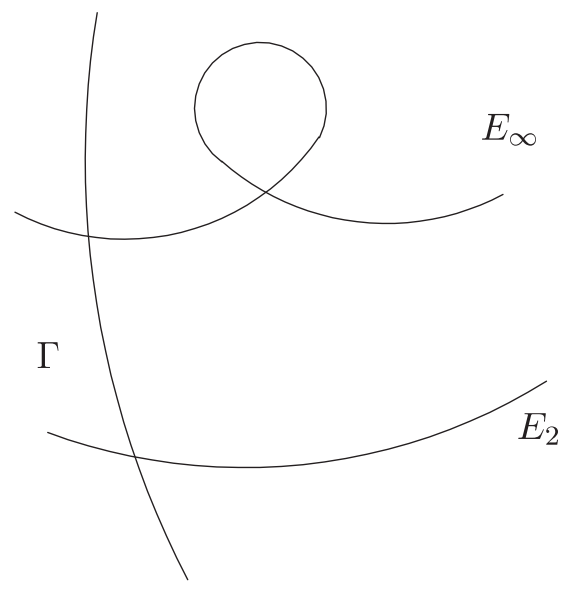

Observe that $L \in \Delta_{0} \cap \Delta_{1}$. Let $\overline{\mathcal{D}_{0}}$ be a component of $\overline{\mathcal{D}} \cap \Delta_{0}$ containing $L$. Since we can assume that the generic element of this component does not belong to $\Delta_{i}$ for $i \geqslant 2$, we obtain the following five cases:

(a) The generic element of $\overline{\mathcal{D}_{0}}$ is an irreducible curve with only one node.

(b) The generic element of $\overline{\mathcal{D}_{0}}$ consists of an irreducible curve with only one node with an elliptic curve attached at a smooth point.

(c) The generic element of $\overline{\mathcal{D}_{0}}$ consists of a smooth irreducible curve with an elliptic nodal curve attached at a smooth point.

(d) The generic element of $\overline{\mathcal{D}_{0}}$ consists of two irreducible smooth curves glued at two different points.

(e) The generic element of $\overline{\mathcal{D}_{0}}$ is an irreducible curve with two nodes.

For topological reasons the cases (d) and (e) cannot occur. Indeed, the number of nodal points in a stable curve such that the curve remains connected when we remove the point cannot decrease under specialization. Since there are two such a points in a generic element of type (d) and (e) and only one in our limit curve $L$, we can ignore these cases.

In this section we will specialize the isogeny $\chi$ to a generic curve of the component $\overline{\mathcal{D}_{0}}$ that contains our limit curve $L$.

Remark 5.1. We will use several times that the limit of a family of isogenies of Jacobians is also an isogeny, that is, an étale surjective map between the generalized Jacobians. We give a brief argument to justify this. Consider two semistable flat families of curves parametrized by disks $\pi^{\prime}: \mathcal{C}^{\prime} \longrightarrow \mathbb{D}$ and $\pi: \mathcal{C} \longrightarrow \mathbb{D}$ such that the curves are smooth away from the central fibers $\mathcal{C}_{0}^{\prime}$ and $\mathcal{C}_{0}$. For a family of isogenies $\varphi: \mathcal{J C}^{\prime} \longrightarrow \mathcal{J C}$ over the punctured disk, the existence of a map 


\section{Marcucci, J. C. Naranjo and G. P. Pirola}

$\varphi_{0}: \mathcal{J C}_{0}^{\prime} \longrightarrow \mathcal{J C}_{0}$ of semiabelian varieties is given in $[\mathrm{BP} 89,(1.2 .6)$ and (1.3.1)]. We want to prove that this map is also an isogeny. Recall that the family of Jacobians is given by

$$
\mathcal{J C}^{\prime}=R^{1} \pi_{*} \mathcal{O} / R^{1} \pi_{*} \mathbb{Z}
$$

and analogously for $\mathcal{J C}$. The fact that $\varphi_{t}$ is an isogeny for $t \neq 0$ implies that there is a sequence over the punctured disk,

$$
0 \longrightarrow R^{1} \pi_{*}^{\prime} \mathbb{Z} \longrightarrow R^{1} \pi_{*} \mathbb{Z} \longrightarrow \tau \longrightarrow 0
$$

where $\tau$ is a torsion local system. Hence, tensoring with $\mathbb{Q}$, we obtain an isomorphism

$$
R^{1} \pi_{*}^{\prime} \mathbb{Q} \cong R^{1} \pi_{*} \mathbb{Q} .
$$

Since the rational cohomology of the central fiber can be identified with the invariant subspace by the action of the monodromy, we have

$$
H^{1}\left(\mathcal{C}_{0}^{\prime}, \mathbb{Q}\right) \cong H^{1}\left(\mathcal{C}_{\mid \mathbb{D}^{*}}^{\prime}, \mathbb{Q}\right)^{\mathrm{inv}} \cong H^{0}\left(\mathcal{C}_{\mid \mathbb{D}^{*}}^{\prime}, R^{1} \pi_{*}^{\prime} \mathbb{Q}\right)
$$

and therefore we conclude that $H^{1}\left(\mathcal{C}_{0}^{\prime}, \mathbb{Q}\right) \cong H^{1}\left(\mathcal{C}_{0}, \mathbb{Q}\right)$. So the map $\varphi_{0}$ is an isogeny between the generalized Jacobians.

When we go to the limit, the information we obtain is different according to the three cases given above.

Case (a). We consider the normalization map $p: \Delta_{0} \rightarrow \rightarrow \mathcal{M}_{g-1}$ restricted to $\overline{\mathcal{D}_{0}}$. There are two possibilities according to the dimension of the generic fiber of $p_{0}:=\left.p\right|_{\overline{\mathcal{D}_{0}}}$.

Case (a.1). Assume that $p_{0}$ is dominant, therefore the generic fiber has dimension $\operatorname{dim} \overline{\mathcal{D}_{0}}-$ $\operatorname{dim} \mathcal{M}_{g-1}=3 g-5-3 g+6=1$. For a generic element $t_{0} \in \overline{\mathcal{D}_{0}}$, the limit map $\chi_{t_{0}}: J C_{t_{0}}^{\prime} \longrightarrow J C_{t_{0}}$ gives a diagram of extensions

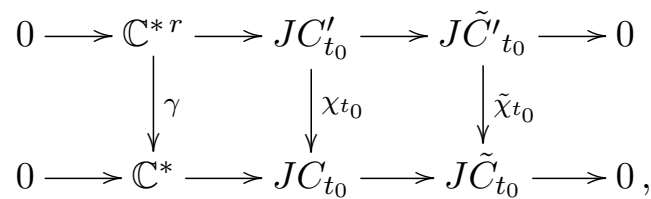

where $\tilde{C}_{t_{0}}^{\prime}$ and $\tilde{C}_{t_{0}}$ stand for the normalizations of $C_{t_{0}}^{\prime}$ and $C_{t_{0}}$, respectively. Since $\chi_{t_{0}}$ has finite kernel, $r$ must be 1 and $\gamma(z)=z^{m}$ for some non-zero integer $m$. Since $g-1 \geqslant 4$, we can apply the main result in [BP89], and we get $\tilde{C}_{t_{0}}^{\prime}=\tilde{C}_{t_{0}}$ and that the isogeny $\tilde{\chi}_{t_{0}}$ is $n$ times the identity. Assume that $C_{t_{0}}$ (respectively, $C_{t_{0}}^{\prime}$ ) is obtained from $\tilde{C}_{t_{0}}$ by pinching two distinct points $p, q$ (respectively, $p^{\prime}, q^{\prime}$ ). As in [BP89, Section 2], to compare the extension classes of each horizontal short exact sequence, we decompose the last diagram into

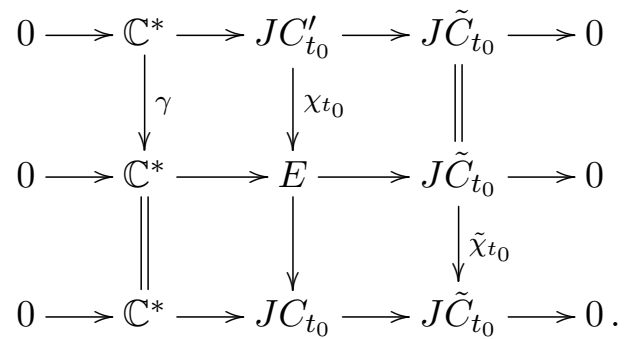




\section{ISOGENIES OF JACOBIANS}

We identify (up to a sign) the extension class

$$
\left[J C_{t_{0}}^{\prime}\right] \in \operatorname{Ext}\left(J \tilde{C}_{t_{0}}, \mathbb{C}^{*}\right) \cong \operatorname{Pic}^{0}\left(J \tilde{C}_{t_{0}}\right) \cong J \tilde{C}_{t_{0}}
$$

with $p^{\prime}-q^{\prime}$ and analogously $\left[J C_{t_{0}}\right]$ with $p-q$. Then the equality $[E]=\gamma_{*}\left(\left[J C_{t_{0}}^{\prime}\right]\right)=\tilde{\chi}_{t_{0}}^{*}\left(\left[J C_{t_{0}}\right]\right)$ provides the following relation in $J \tilde{C}_{t_{0}}$ :

$$
n(p-q)= \pm m\left(p^{\prime}-q^{\prime}\right) .
$$

Hence we can assume that $n p+m q^{\prime}=m p^{\prime}+n q$ in $\operatorname{Pic}\left(\tilde{C}_{t_{0}}\right)$. We also assume that the points are different. Since the dimension of the generic fiber of $p_{0}$ is 1 , we have a 1-dimensional family of maps $\tilde{C}_{t_{0}} \longrightarrow \mathbb{P}^{1}$ of degree $n+m$ with two fibers as above. The Riemann-Hurwitz theorem implies that

$$
2 g\left(\tilde{C}_{t_{0}}\right)-2=2 g-4=(n+m)\left(2 g\left(\mathbb{P}^{1}\right)-2\right)+2(n-1)+2(m-1)+r=-4+r,
$$

so the number $r$ of ramification points out of the special fibers $n p+m q^{\prime}$ and $m p^{\prime}+n q$ is $r=2 g$. Then the Hurwitz scheme of maps of degree $n+m$ into $\mathbb{P}^{1}$ with $r+2=2 g+2$ discriminant points must cover $\mathcal{M}_{g-1}$ with generic fibers of dimension 1. Comparing dimensions, we obtain

$$
2 g+2-\operatorname{dim} \operatorname{Aut}\left(\mathbb{P}^{1}\right)-\operatorname{dim} \text { generic fiber }=2 g+2-4=2 g-2 \geqslant \operatorname{dim} \mathcal{M}_{g-1}=3 g-6,
$$

which contradicts the hypothesis $g \geqslant 5$. Hence the extension is the same and $n=m$.

Case (a.2). Assume that the generic fiber of $p_{0}$ has dimension 2. As before we get a diagram

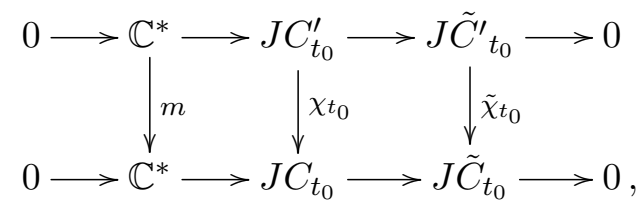

but now we do not have the genericity of $\tilde{C}_{t_{0}}$, so we cannot directly apply the main result in [BP89]. The relation between extension classes is in this case

$$
m\left(p^{\prime}-q^{\prime}\right)=\tilde{\chi}_{t_{0}}^{*}(p-q),
$$

in $J \tilde{C}_{t_{0}}^{\prime}$. In other words, the isogeny $\tilde{\chi}_{t_{0}}$ induces a map between the surfaces $\tilde{C}_{t_{0}}-\tilde{C}_{t_{0}}$ and $m\left(\tilde{C}_{t_{0}}^{\prime}-\tilde{C}_{t_{0}}^{\prime}\right)$. By using the arguments of [BP89, Section 3], one easily checks that, as before, the curves are the same and the map is the multiplication by an integer. So we have proved the following result.

Proposition 5.2. Let $\chi: \mathcal{J}^{\prime} \longrightarrow \mathcal{J}$ be a family of isogenies parametrized by $\mathcal{D}$, and let $\chi_{t_{0}}: J C_{t_{0}}^{\prime} \longrightarrow J C_{t_{0}}$ be a specialization to a generic point $t_{0}$ of a component of the boundary $\overline{\mathcal{D}} \cap \Delta_{0}$, where the curve $C_{t_{0}}$ is an irreducible curve with only one node. Then $C_{t_{0}}^{\prime} \cong C_{t_{0}}$ and $\chi_{t_{0}}$ is the multiplication by a non-zero integer.

Case (b). We now assume that the limit curve $L$ belongs to an irreducible component $\overline{\mathcal{D}_{0}}$ of $\overline{\mathcal{D}} \cap \Delta_{0}$ whose generic element consists of a smooth curve $\Gamma$ of genus $g-1$ and a nodal elliptic curve $E_{\infty}$ (that is, a $\mathbb{P}^{1}$ with the points 0 and 1 identified) glued to $\Gamma$ at a point $p \in \Gamma$. As above, we denote by $C_{t_{0}}=\Gamma \cup_{p} E_{\infty}$ a generic curve in $\overline{\mathcal{D}_{0}}$. Observe that the natural map $\overline{\mathcal{D}_{0}} \rightarrow-\mathcal{M}_{g-1}$ must be dominant by a count of dimensions (the fiber has dimension at most 1); therefore we can assume that $\Gamma$ is generic in $\mathcal{M}_{g-1}$.

We consider the specialization of the family of isogenies to our curve $\chi_{t_{0}}: J C_{t_{0}}^{\prime} \longrightarrow J C_{t_{0}}=$ 


\section{Marcucci, J. C. Naranjo and G. P. Pirola}

$J \Gamma \times \mathbb{C}^{*}$ which fits in a diagram of extensions

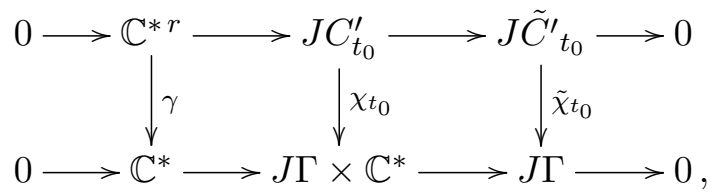

where $\tilde{C}_{t_{0}}^{\prime}$ stands for the normalization of $C_{t_{0}}^{\prime}$. Since $\chi_{t_{0}}$ has finite kernel, $r$ must be 1 and $\gamma(z)=z^{m}$ for some non-zero integer $m$. Since $g-1 \geqslant 4$, we can apply the main result in [BP89], and we get $\tilde{C}_{t_{0}}^{\prime}=\Gamma$ and that the isogeny $\tilde{\chi}_{t_{0}}$ is $n$ times the identity. So the diagram above becomes

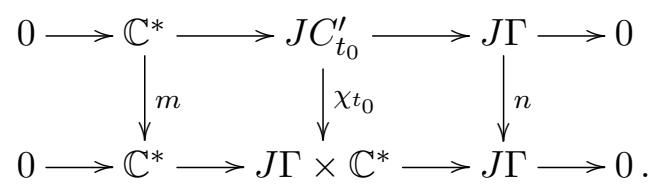

Since the extension class of the first row corresponds to a generalized Jacobian, there exist points $q_{1}, q_{2} \in \Gamma$ such that this class corresponds (up to a sign) to $q_{1}-q_{2} \in J \Gamma$. Therefore, since the class of the second row is zero, we get $m\left(q_{1}-q_{2}\right)=0$.

Moving the point $p$ in $\Gamma$, we have a positive-dimensional family of pairs of points $\left(q_{1}, q_{2}\right) \in$ $C \times C$ with this property. This family has to be the diagonal since the map $\Gamma \times \Gamma \longrightarrow J \Gamma$ has degree 1 and finite fibers out of the diagonal. Hence we obtain $q_{1}=q_{2}$.

Hence the extension given by the first row is also trivial: $J C_{t_{0}}^{\prime} \cong J \Gamma \times \mathbb{C}^{*}$ and $\chi_{t_{0}}=\left(\begin{array}{cc}n & 0 \\ 0 & m\end{array}\right)$. We get the following result.

Proposition 5.3. Let $\chi: \mathcal{J}^{\prime} \longrightarrow \mathcal{J}$ be a family of isogenies parametrized by $\mathcal{D}$, and let $\chi_{t_{0}}: J C_{t_{0}}^{\prime} \longrightarrow J C_{t_{0}}$ be a specialization to a generic point $t_{0}$ of a component of the boundary $\overline{\mathcal{D}} \cap \Delta_{0}$, where the curve $C_{t_{0}}$ is a reducible curve consisting of a smooth curve of genus $g-1$ with a nodal elliptic curve $E_{\infty}$ attached at one point. Then $C_{t_{0}}^{\prime} \cong C_{t_{0}}$ and the isogeny in the compact part is the multiplication by a non-zero integer.

Case (c). Finally, we assume that the limit curve $L$ belongs to an irreducible component $\overline{\mathcal{D}_{0}}$ of $\overline{\mathcal{D}} \cap \Delta_{0}$ whose generic element consists of a nodal curve $\Gamma_{0}$ of genus $g-1$ and an elliptic curve $E$ glued to $\Gamma_{0}$ at a smooth point $p \in \Gamma_{0}$. As above, we denote by $C_{t_{0}}=\Gamma_{0} \cup_{p} E$ a generic curve in $\overline{\mathcal{D}_{0}}$. Observe that the natural map $\overline{\mathcal{D}_{0}} \rightarrow \Delta_{0}\left(\mathcal{M}_{g-1}\right)$ must be dominant (here $\Delta_{0}\left(\mathcal{M}_{g-1}\right)$ denotes the $\Delta_{0}$ divisor in the moduli space $\left.\mathcal{M}_{g-1}\right)$. Indeed, the generic fiber of this map has dimension at most 2 and is parametrized by the smooth point $p$ in $\Gamma_{0}$ and the moduli of the elliptic curves. Since $\operatorname{dim} \overline{\mathcal{D}_{0}}=3 g-5$ and $\operatorname{dim} \Delta_{0}\left(\mathcal{M}_{g-1}\right)=3 g-7$, the dominance follows. Therefore we can assume that $\Gamma_{0}$ is generic in $\Delta_{0}\left(\mathcal{M}_{g-1}\right)$, and also that its normalization $\tilde{\Gamma}_{0}$ is generic in $\mathcal{M}_{g-2}$. Moreover, all the curves $\Gamma_{0} \cup_{p} E$ are contained in $\overline{\mathcal{D}_{0}}$ for a generic $\Gamma_{0}$.

As in the other cases, we consider the specialization of the family of isogenies to our curve $\chi_{t_{0}}: J C_{t_{0}}^{\prime} \longrightarrow J C_{t_{0}}=J \Gamma_{0} \times E$ which fits in a diagram of extensions

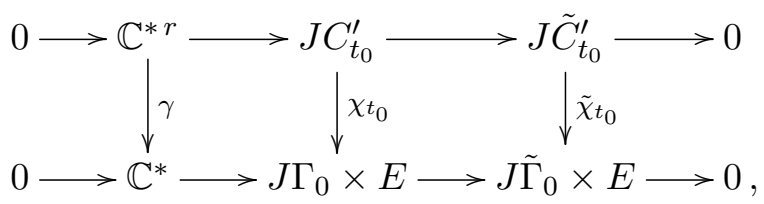

where $\tilde{C}_{t_{0}}^{\prime}$ stands for the normalization of $C_{t_{0}}^{\prime}$. Since $\chi_{t_{0}}$ has finite kernel, $r$ must be 1 and 


\section{ISOGENIES OF JACOBIANS}

$\gamma(z)=z^{m}$ for some non-zero integer $m$.

We claim that $J \tilde{C}_{t_{0}}^{\prime}$ must be a product of Jacobians (in other words, the smooth curve $\tilde{C}_{t_{0}}^{\prime}$ is reducible). We prove this by contradiction. Assume that $\tilde{C}_{t_{0}}^{\prime}$ is irreducible and compare the extension classes. This gives (up to a sign) a relation $m\left(p^{\prime}-q^{\prime}\right)=\tilde{\chi}_{t_{0}}(p-q)$ in $J \tilde{C}_{t_{0}}^{\prime}$. Moving the points $p$ and $q$ in the fixed curve $\tilde{\Gamma}_{0}$, we get that the image of $\tilde{\Gamma}_{0}-\tilde{\Gamma}_{0}$ by the isogeny is the surface $\tilde{C}_{t_{0}}^{\prime}-\tilde{C}_{t_{0}}^{\prime}$, which is impossible since it is contained in the proper abelian subvariety $\tilde{\chi}_{t_{0}}^{*}\left(J \tilde{\Gamma}_{0}\right)$.

By the genericity of $\tilde{\Gamma}_{0}$, we can assume that $J \tilde{\Gamma}_{0}$ is simple. Then $J \tilde{C}_{t_{0}}^{\prime} \cong J \tilde{C}_{t_{0}}^{\prime \prime} \times E^{\prime}$, where $\tilde{C}_{t_{0}}^{\prime \prime}$ is an irreducible curve of genus $g-2$ and $E^{\prime}$ stands for a smooth elliptic curve. So $C_{t_{0}}^{\prime}$ is a nodal curve $C_{t_{0}}^{\prime \prime}$ with the elliptic curve attached at a smooth point. The extension class is the difference of two points $p^{\prime \prime}, q^{\prime \prime} \in \tilde{C}_{t_{0}}^{\prime \prime}$. The diagram above becomes

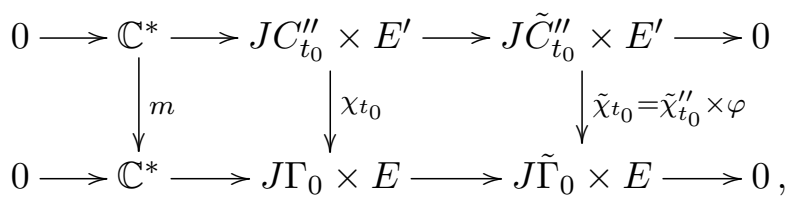

where $\varphi: E^{\prime} \longrightarrow E$ is a non-constant map of elliptic curves. The relation between extension classes is in this case (up to a sign)

$$
m\left(p^{\prime \prime}-q^{\prime \prime}\right)=\left(\tilde{\chi}_{t_{0}}^{\prime \prime}\right)^{*}(p-q)
$$

in $J \tilde{C}_{t_{0}}^{\prime \prime}$. In other words, the isogeny $\tilde{\chi}_{t_{0}}^{\prime \prime}$ induces a map between the surfaces $\tilde{\Gamma}_{0}-\tilde{\Gamma}_{0}$ and $m\left(\tilde{C}_{t_{0}}^{\prime \prime}-\tilde{C}_{t_{0}}^{\prime \prime}\right)$. By using the arguments of [BP89, Section 3] one easily checks the following facts: the curves $C_{t_{0}}^{\prime \prime}$ and $\Gamma_{0}$ are isomorphic, the isogeny $\tilde{\chi}_{t_{0}}^{\prime \prime}$ is a non-zero multiple $n$ of the identity, $n=m$, and then $\chi_{t_{0}}=n \times \varphi$.

Proposition 5.4. Let $\chi: \mathcal{J}^{\prime} \longrightarrow \mathcal{J}$ be a family of isogenies parametrized by $\mathcal{D}$, and let $\chi_{t_{0}}: J C_{t_{0}}^{\prime} \longrightarrow J C_{t_{0}}$ be a specialization to a generic point $t_{0}$ of a component of the boundary $\overline{\mathcal{D}} \cap \Delta_{0}$, where the curve $C_{t_{0}}$ is a reducible curve consisting of a nodal curve $\Gamma_{0}$ of genus $g-1$ with an elliptic curve $E$ attached at one smooth point. Then $C_{t_{0}}^{\prime}$ also is of the form $\Gamma_{0} \cup_{p} E^{\prime}$ for some elliptic curve $E^{\prime}$, and the isogeny induces the multiplication by $n$ on $J \Gamma_{0}$.

\section{End of the proof}

Let us go back to our family of isogenies $\chi: \mathcal{J}^{\prime} \longrightarrow \mathcal{J}$ parametrized by (some covering of) the divisor $\mathcal{D}$.

Consider a generic point $t \in \mathcal{D}$ corresponding to smooth curves $C_{t}^{\prime}$ and $C_{t}$. Observe that for all $t$, the isogeny is determined by the map at the level of homology groups,

$$
\chi_{t, \mathbb{Z}}: H_{1}\left(C_{t}^{\prime}, \mathbb{Z}\right) \longrightarrow H_{1}\left(C_{t}, \mathbb{Z}\right),
$$

which we still denote by $\chi_{t}$. We set $\Lambda_{t} \subset H_{1}\left(C_{t}, \mathbb{Z}\right)$ for the image of $\chi_{t}$. This is a sublattice of maximal rank $2 g$. We first note that the proof of [BP89, Proposition 4.2.1] applies verbatim to obtain the following result.

Proposition 6.1. Assume that $\Lambda_{t}=n H_{1}\left(C_{t}, \mathbb{Z}\right)$ for some positive integer $n$. Then $C_{t}^{\prime} \cong C_{t}$ and $\chi_{t}$ is the multiplication by $n$.

Therefore to finish the proof of the theorem, we have to show the equality $\Lambda_{t}=n H_{1}\left(C_{t}, \mathbb{Z}\right)$. To do this, the main idea is to get information on $\Lambda_{t}$ from the homology groups of some convenient 


\section{Marcucci, J. C. Naranjo and G. P. Pirola}

limits $C_{0}$. In the previous sections we have shown the existence of certain limits and we have seen in Propositions 5.2, 5.3 and 5.4 what the image of the limit of $\chi_{t}$ is when $t$ goes to one of these degenerations.

In order to get information on the lattice $\Lambda_{t}$ of the generic smooth curve $C_{t}$ from the geometry of some limit curve $C_{0}$, we use the following principle: We can assume that there exists a disk $\mathbb{D} \subset \overline{\mathcal{D}} \subset \overline{\mathcal{M}_{g}}$ centered at the class of the curve $C_{0}$ such that the curves $C_{t}, C_{t}^{\prime}$ corresponding to $\mathbb{D} \backslash\{0\}$ are smooth. After performing a base change (that we skip to simplify the notation), we can assume that there is a family of isogenies $\chi_{\mathbb{D}}: \mathcal{J}_{\mathbb{D}}^{\prime} \longrightarrow \mathcal{J}_{\mathbb{D}}$ that coincides with the original isogeny $\chi_{t}$ for a generic $t$. We denote by $\chi_{0}: J C_{0}^{\prime} \longrightarrow J C_{0}$ the limit isogeny. We call $\mathcal{C}_{\mathbb{D}}^{\prime}$ and $\mathcal{C}_{\mathbb{D}}$ the corresponding families of curves. Since the central fibers $C_{0}^{\prime}$ and $C_{0}$ are retracts of $\mathcal{C}_{\mathbb{D}}^{\prime}$ and $\mathcal{C}_{\mathbb{D}}$, respectively, we have a diagram as follows:

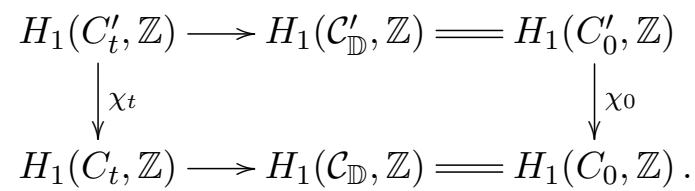

Let us first consider the simplest case. Assume that we are in case (a) and that $C_{0}$ is a generic element of $\overline{\mathcal{D}} \cap \Delta_{0}$, so it is an irreducible curve with only one node. Remember that $C_{0}$ can be degenerated in this component to a curve $L$ consisting of a curve of genus $g-2$ with two attached curves, one elliptic (called $E_{2}$ ) and the other nodal and rational $\left(E_{\infty}\right)$. By Proposition 5.2, we have $C_{0}^{\prime} \cong C_{0}$ and $\chi_{0}=n \cdot \mathrm{Id}$. The kernels of the horizontal maps are generated by the vanishing cycles $a_{1}^{\prime}$ and $a_{1}$, respectively; therefore $\chi_{t}\left(a_{1}^{\prime}\right)=l a_{1}$ for some $l$. On the other hand, we can lift a basis in $H_{1}\left(C_{0}, \mathbb{Z}\right)$ and construct simplectic bases $a_{1}^{\prime}, b_{1}^{\prime}, \ldots, a_{g}^{\prime}, b_{g}^{\prime}$ in $H_{1}\left(C_{t}^{\prime}, \mathbb{Z}\right)$ and $a_{1}, b_{1}, \ldots, a_{g}, b_{g}$ in $H_{1}\left(C_{t}, \mathbb{Z}\right)$ in such a way that

$$
\chi_{s}\left(a_{i}^{\prime}\right)=n a_{i}+s_{i} a_{1}, \quad \chi_{t}\left(b_{1}^{\prime}\right)=n b_{1}+t_{1} a_{1}, \quad \chi_{t}\left(b_{i}^{\prime}\right)=n b_{i}+t_{i} a_{1}
$$

for some integers $s_{i}$ and $t_{i}$ and $i \geqslant 2$. We can also assume that $a_{g}, b_{g}$ correspond to cycles which become a basis of the homology of $E_{2} \subset L$.

By the genericity of the curve $C_{t}$ in a divisor of the moduli space, the pull-back of the theta divisor is a multiple of the theta divisor in $J C_{t}^{\prime}$. This translates into the existence of a non-zero integer $m$ such that the cup-product satisfies

$$
\chi_{t}(x) \cup \chi_{t}(y)=m x \cup y .
$$

Then we obtain

$$
\begin{aligned}
& m=\chi_{t}\left(a_{1}^{\prime}\right) \cup \chi_{t}\left(b_{1}^{\prime}\right)=l a_{1} \cup\left(n b_{1}+t_{1} a_{1}\right)=l n, \\
& m=\chi_{t}\left(a_{2}^{\prime}\right) \cup \chi_{t}\left(b_{2}^{\prime}\right)=n^{2},
\end{aligned}
$$

so $n=l$. With similar computations it is easy to prove that $s_{i}=t_{i}=0$ for $i \geqslant 2$, hence

$$
\chi_{t}\left(a_{1}^{\prime}\right)=n a_{1}, \quad \chi_{t}\left(a_{i}^{\prime}\right)=n a_{i}, \quad \chi_{t}\left(b_{i}^{\prime}\right)=n b_{i} \quad \text { for } \quad i \geqslant 2 .
$$

To get the piece of information which is still unknown, we need to consider a second limit. We saw in Corollary 4.7 that by fixing $\Gamma$, we can move the two elliptic curves $E_{1}$ and $E_{2}$ freely; that is, $E_{1} \cup \Gamma \cup E_{2} \in \overline{\mathcal{D}}$ for all $E_{1}, E_{2}$. We select a second limit curve $\hat{L}=E_{1} \cup \Gamma \cup E_{\infty}$ in such a way that the corresponding vanishing cycle is now $a_{g}$. Again, to simplify, we assume that $\hat{L}$ belongs to case (a) of Section 5. Then, with the same argument, we get that $\chi_{t}$ satisfies (for the same simplectic basis)

$$
\chi_{t}\left(a_{i}^{\prime}\right)=\hat{n} a_{i}, \quad \chi_{t}\left(b_{i}^{\prime}\right)=\hat{n} b_{i} \quad \text { for } i \leqslant g-1
$$


and $\chi_{t}\left(a_{g}^{\prime}\right)=\hat{n} a_{g}$. Therefore $\hat{n}=n$ and $\Lambda_{t}=n H_{1}\left(C_{t}, \mathbb{Z}\right)$ for all $s \neq 0$. Hence we have finished (under the assumption that $L$ and $\hat{L}$ are limit curves of nodal curves). The rest of the proof consists of the description of the small modifications that have to be done to take care of the rest of the cases. Observe that the information on the limit given in case (b) (see Proposition 5.3) is the same as that given in case (a); that is, we again have the relations (6.1). So we only have to take care of the situation when at least one of the limit curves $L$ and $\hat{L}$ belongs to case (c). Assume, for example, that $L$ does. Using the first limit as above, we know that

$$
\chi_{t}\left(a_{1}^{\prime}\right)=n a_{1}, \quad \chi_{t}\left(a_{i}^{\prime}\right)=n a_{i}, \quad \chi_{t}\left(b_{i}^{\prime}\right)=n b_{i} \quad \text { for } \quad 2 \leqslant i \leqslant g-1 .
$$

The difference with the previous cases is that we have no control over $\chi_{t}\left(b_{1}^{\prime}\right)$. Remember that $L$ is the limit of a curve $\Gamma_{0} \cup E$, where $\Gamma_{0}$ is a nodal curve intersecting the elliptic curve $E$ in a smooth point. Denoting by $\tilde{\Gamma}_{0}$ the normalization of $\Gamma_{0}$, we note that all nodal curves with this normalization belong to the divisor $\overline{\mathcal{D}_{0}}$. So we freely change the node in such a way that the vanishing cycle of the node becomes $a_{2}$ (instead of $a_{1}$ ). By using the two vanishing cycles we get $\chi_{t}\left(b_{1}^{\prime}\right)=n b_{1}^{\prime}$, so we again recover the relations (6.1). This finishes the proof of the theorem.

Our theorem can be interpreted as a type of Noether-Lefschetz problem in the following way: Consider in $\mathcal{M}_{g} \times \mathcal{M}_{g}$ the set

$$
\mathcal{N} \mathcal{L}_{g}=\left\{\left(C^{\prime}, C\right) \mid \operatorname{rank} N S\left(C \times C^{\prime}\right) \geqslant 3\right\} .
$$

A consequence of what we have proved is the following result.

Corollary 6.2. For $g \geqslant 5$, all components of $\mathcal{N} \mathcal{L}_{g}$ outside the diagonal have dimension less than or equal to $3 g-5$.

The first natural problem one could face in this context is to investigate the existence of dimension 10 components in $\mathcal{N}_{5}$. Similar problems on isogenies can be considered for other families of abelian varieties (see for example [NP94]).

\section{ACKNOWLEDGEMENTS}

We thank Gavril Farkas for useful and stimulating conversations on the moduli space of curves.

\section{REFERENCES}

ACG11 E. Arbarello, M. Cornalba and P. A. Griffiths, Geometry of algebraic curves. Vol. II, Grundlehren Math. Wiss., vol. 268 (Springer, Heidelberg, 2011); http://dx.doi.org/10. 1007/978-3-540-69392-5.

ACGH85 E. Arbarello, M. Cornalba, P. A. Griffiths and J. Harris, Geometry of algebraic curves. Vol. I, Grundlehren Math. Wiss., vol. 267 (Springer-Verlag, New York, 1985); http://dx.doi.org/ 10.1007/978-1-4757-5323-3.

BP89 F. Bardelli and G.P. Pirola, Curves of genus g lying on a g-dimensional Jacobian variety, Invent. Math. 95 (1989), no. 2, 263-276; http://dx.doi.org/10.1007/BF01393898.

CGGH83 J. Carlson, M. Green, P. Griffiths and J. Harris, Infinitesimal variations of Hodge structure. I, Compos. Math. 50 (1983), no. 2-3, 109-205; http://www. numdam.org/item?id=CM_1983__50_ 2-3_109_0.

CGT92 C. Ciliberto, G. van der Geer and M. Teixidor i Bigas, On the number of parameters of curves whose Jacobians possess nontrivial endomorphisms, J. Algebraic Geom. 1 (1992), no. 2, 215229. 


\section{Marcucci, J. C. Naranjo and G. P. Pirola}

CM91 M. Coppens and G. Martens, Secant spaces and Clifford's theorem, Compos. Math. 78 (1991), no. 2, 193-212; http://www. numdam.org/item?id=CM_1991__78_2_193_0.

Mum70 D. Mumford, Abelian varieties, Tata Inst. Fund. Res. Stud. Math., vol. 5 (Oxford University Press, London, 1970).

NP94 J. C. Naranjo and G.P. Pirola, On the genus of curves in the generic Prym variety, Indag. Math. (N.S.) 5 (1994), no. 1, 101-105; http://dx.doi.org/10.1016/0019-3577 (94) 90036-1.

Pir88 G. P. Pirola, Base number theorem for abelian varieties. An infinitesimal approach, Math. Ann. 282 (1988), no. 3, 361-368; http://dx.doi.org/10.1007/BF01460039.

Ran86 Z. Ran, On a theorem of Martens, Rend. Sem. Mat. Univ. Politec. Torino 44 (1986), no. 2, $287-291$.

Valeria Marcucci valeria.marcucci@unipv.it

Università degli Studi di Pavia, Dipartimento di Matematica, Via Ferrata, 1, 27100 Pavia, Italy

Juan Carlos Naranjo jenaranjo@ub.edu

Universitat de Barcelona, Facultat de Matemàtiques, Gran Via 585, 08007 Barcelona, Spain

Gian Pietro Pirola gianpietro.pirola@unipv.it

Università degli Studi di Pavia, Dipartimento di Matematica, Via Ferrata, 1, 27100 Pavia, Italy 